Scientia Agricola

versão impressa ISSN 0103-9016

Sci. agric. v.57 n.1 Piracicaba jan./mar. 2000

doi: $10.1590 / S 0103-90162000000100023$

\title{
Alumínio e a absorção de cálcio por mudas de pimenta do reino
}

\author{
Carlos Alberto Costa Veloso ${ }^{1 *}$; Eurípedes \\ Malavolta2; Takashi Muraoka2; Eduardo Jorge \\ Maklouf Carvalho' \\ ${ }^{1}$ Embrapa/Amazônia Oriental, C.P. 48 - CEP: 66.095-100 - Belém, PA. \\ ${ }^{2}$ USP/CENA, C.P. 96 - CEP: 13400-970 - Piracicaba, SP. \\ *Autor correspondente<veloso@cpatu.embrapa.br>
}

RESUMO: Um experimento com solução nutritiva foi conduzido em casa de vegetação com o objetivo de verificar os efeitos da nutrição de cálcio na toxicidade de alumínio em mudas de pimenta do reino. Os tratamentos constituíram-se de três concentrações de cálcio (10; 40 e $160 \mathrm{mg} \mathrm{L}^{-1}$ ) adicionados como CaCl 2 em combinação com três concentrações de alumínio $\left(0 ; 10\right.$ e $20 \mathrm{mg} \mathrm{L}{ }^{-1}$ ) adicionados como $\mathrm{AlCl}_{3} \cdot 6 \mathrm{H}_{2} \mathrm{O}$. A solução nutritiva continha as seguintes concentrações dos íons expressos em mg L-1: N- $\mathrm{NO}_{3}(80,5) ; \mathrm{N}-\mathrm{NH}_{4}(10,5) ; \mathrm{P}(1,5)$; $\mathrm{K}(39,0) ; \mathrm{Mg}(24,0) ; \mathrm{S}-\mathrm{SO}_{4}(32,0) ; \mathrm{B}(0,5) ; \mathrm{Cu}(0,06) ; \mathrm{Fe}(5,0) ; \mathrm{Mn}(1,0) ; \mathrm{Mo}(0,03)$ e Zn(0,10). Os tratamentos com alumínio retardaram o crescimento das mudas de pimenta do reino, induziram sintomas de toxicidade de alumínio na parte aérea e principalmente aumentaram o diâmetro das raízes. O acúmulo de cálcio aumentou com o incremento da concentração de cálcio na solução na ausência de alumínio, nas raízes o conteúdo de cálcio foi diminuído com o aumento da concentração de alumínio na solução. A absorção de alumínio diminuiu com o aumento da concentração de cálcio na solução.

Palavras-chave: Piper nigrum, toxicidade de alumínio, cálcio, nutrição

\section{Aluminum and calcium absorption by black pepper seedlings}

ABSTRACT: A nutrient solution experiment with was carried out in a greenhouse with the objective of verifying the specific effects of calcium and aluminum toxicity on black pepper seedlings. The treatments were set up in three concentrations of calcium (10; 40 and $160 \mathrm{mg} \mathrm{L}^{-1}$ ) added as $\mathrm{CaCl}_{2}$ in combination with three concentrations of aluminum $\left(0 ; 10\right.$ and $\left.20 \mathrm{mg} \mathrm{L}^{-1}\right)$ added as $\mathrm{AlCl}_{3} .6 \mathrm{H}_{2} \mathrm{O}$. The nutrient solution contained the following ion concentrations in $\mathrm{mg} \mathrm{L}^{-1}: \mathrm{N}-\mathrm{NO}_{3}(80,5) ; \mathrm{N}-\mathrm{NH}_{4}(10,5) ; \mathrm{P}(1,5) ; \mathrm{K}(39,0) ; \mathrm{Mg}(24,0) ; \mathrm{S}-\mathrm{SO}(32,0) ; \mathrm{B}(0,5)$; $\mathrm{Cu}(0,06) ; \mathrm{Fe}(5,0) ; \mathrm{Mn}(1,0) ; \mathrm{Mo}(0,03)$ and $\mathrm{Zn}(0,10)$. The treatments with aluminum reduced black pepper growth and the diameter of roots increased. Calcium absorption increased with the increase of calcium concentration in the solution, in the absence of aluminum. Calcium absorption by roots decreased with the increase of the concentration of aluminum in the solution. Aluminum absorption decreased with the increase of the concentration of calcium in the solution.

Key words: Piper nigrum, aluminum of toxicity, calcium, nutrition

\section{INTRODUÇÃO}

O cultivo da pimenta do reino na região amazônica é realizado geralmente em solos de baixa fertilidade natural, caracterizados por apresentar baixa saturação por bases e, frequentemente, possuir alumínio trocável em quantidades altas que limitam o crescimento de raízes de muitas espécies cultivadas (Veloso et al., 1995). Nestas condições, as raízes são incapazes de explorar maior volume de solo, a menos que este tenha recebido adubação e calagem para suprir cálcio e outros nutrientes e reduzir as concentrações tóxicas de alumínio (Pavan, 1982).

O efeito nocivo do alumínio limita o crescimento da maioria das plantas cultivadas. A pimenteira do reino é uma planta que parece ser tolerante a solos ácidos, porém praticamente, são raros os trabalhos referentes à calagem e sobretudo às relações cálcio e alumínio nesta cultura. De modo geral, a toxicidade de alumínio em plantas tem sido associada com uma reduzida absorção e translocação de cálcio (Foy et al., 1969). Pavan (1982),

trabalhando com mudas de cafeeiro, verificou que o aumento da concentração de cálcio em solução de 0,05 para $1,25 \mathrm{mmol} . \mathrm{dm}^{-3}$ não diminuiu a atividade dos íons $\mathrm{Al}^{3+}$ para um nível não tóxico. O autor também observou que a 
absorção de cálcio pelas raízes foi progressivamente diminuída com o aumento da concentração de alumínio na solução.

A ocorrência de toxicidade de alumínio em plantas cultivadas é freqüente em muitos solos brasileiros e, na maioria das vezes, está associada aos solos lixiviados, com baixa fertilidade e de elevada acidez. A calagem é a técnica normalmente adotada para contornar tal problema, mas a incorporação do calcário em horizontes subsuperficiais é difícil.

O efeito nocivo de alumínio em plantas tem sido extensivamente pesquisado por muitos cientistas, como Foy et al. (1978), Pavan \& Bingham (1982), Cambraia (1989) e Foy (1992). Geralmente, o efeito tóxico do Al é notado em raízes de plantas antes que qualquer sintoma possa ser evidente na parte aérea. Os sintomas de toxidez são também associados à deficiência de fósforo e com reduzida absorção e translocação de cálcio (Foy, 1974). O excesso de alumínio inibe o crescimento normal de raízes, tornando-as engrossadas, com coloração marrom, menos ramificadas, quebradiças e ocasionalmente com manchas necróticas (Foy, 1992).

Na prática, o uso de corretivos da acidez diminui o efeito tóxico do alumínio. Há inúmeros resultados na literatura demonstrando que através da calagem dos solos ácidos elimina-se o Al trocável e aumenta-se a produção dos cultivos (Pratt, 1966; Lopes, 1984; Malavolta \& Kliemann, 1985). Entretanto, a recomendação dessa prática para a pimenteira do reino tem sido feita de forma emprírica, sem ênfase ao aprofundamento do sistema radicular. Assim, são indicadas as aplicações de pequenas doses na cova de plantio (Albuquerque et al., 1989).

Desta maneira, conduziu-se em casa de vegetação um experimento com plantas de pimenta-do-reino em solução nutritiva, com o objetivo de verificar o efeito da toxicidade do alumínio na absorção de cálcio por mudas de pimenta do reino.

\section{MATERIAL E MÉTODOS}

O trabalho foi realizado no Centro de Energia Nuclear na Agricultura (CENA), da Universidade de São Paulo, em Piracicaba-SP, no período compreendido entre abril a novembro de 1992.

Foram utilizadas mudas de pimenteira do reino (Piper nigrum, L.) cultivar Guajarina, obtidas de estacas herbáceas com dois entre-nós e providas de uma folha no nó superior, coletadas em uma área de plantio comercial no município de Mirassolândia-SP.

Após 120 dias do início do enraizamento, as mudas foram retiradas do substrato (solo), as raízes lavadas com jato de água de torneira e, em seguida, imersas em água desmineralizada para completar a limpeza. O material foi selecionado, procurando-se uniformizar, ao máximo, através da escolha de plantas com a parte aérea e o sistema radicular com padrão de crescimento semelhante. As mudas foram então transferidas para vasos de plástico com capacidade de 2,5 litros e fixadas na tampa pelo caule com espuma de plástico, usando-se uma planta por recipiente.

Durante os primeiros 15 dias após o transplante, as plantas foram mantidas em solução nutritiva completa, diluída a $1 / 4$ da concentração usual; nas duas semanas seguintes receberam solução diluída a $1 / 2$ e em seguida foram submetidas aos tratamentos de acordo com a metodologia usada por Waard (1969) modificada em relação à concentração de fósforo, que foi reduzida para $1,5 \mathrm{mg} \mathrm{L}^{-1}$ para evitar a precipitação do alumínio, cuja composição encontra-se na TABELA 1.

\begin{tabular}{lc} 
TABELA 1 - Composição da solução nutritiva usada para o estu- \\
$\begin{array}{l}\text { do de aluminio e calcio, modificada a partir de Waard } \\
(1969) .\end{array}$ \\
\hline Nutrientes & Concentração \\
\hline & $\left(\mathrm{mg} \mathrm{L}^{-1}\right)$ \\
$\mathrm{N}^{-N \mathrm{NO}_{3}}$ & 80,50 \\
$\mathrm{~N}^{-\mathrm{NH}_{4}}$ & 10,50 \\
$\mathrm{P}$ & 1,50 \\
$\mathrm{~K}$ & 39,00 \\
$\mathrm{Mg}$ & 24,00 \\
$\mathrm{~S}-\mathrm{SO}_{4}$ & 32,00 \\
$\mathrm{~B}$ & 0,50 \\
$\mathrm{Cu}$ & 0,06 \\
$\mathrm{Fe}$ & 5,00 \\
$\mathrm{Mn}$ & 1,00 \\
$\mathrm{Mo}$ & 0,03 \\
$\mathrm{Zn}$ & 0,10 \\
\hline
\end{tabular}

As soluções nutritivas foram renovadas a cada duas semanas e o seu volume completado com água destilada diariamente. $\mathrm{O}$ pH das soluções foi mantido em 4,0 $\pm 0,2$ com adições de $\mathrm{HCl}$ ou $\mathrm{NaOH}$.

O delineamento experimental utilizado foi inteiramente casualizado, em esquema fatorial $3 \times 3$, com três repetições. A unidade experimental foi constituída por um vaso, contendo uma planta.

Os tratamentos constituíram-se de três concentrações de cálcio $\left(10 ; 40\right.$ e $\left.160 \mathrm{mg} \mathrm{L}{ }^{-1}\right)$ adicionados como $\mathrm{CaCl}_{2}$ em combinação com três concentrações de alumínio $\left(0 ; 10\right.$ e $20 \mathrm{mg} \mathrm{L}^{-1}$ ) adicionados como $\mathrm{AlCl}_{3} .6 \mathrm{H}_{2} \mathrm{O}$.

As mudas de pimenteira do reino foram submetidas aos tratamentos por 150 dias, quando foram colhidas. Foram determinados o comprimento das raízes e a massa da matéria seca das folhas, caule e raízes. As amostras foram 
separadas a parte aérea (folhas e caules) e raízes, sendo a secagem a $65^{\circ} \mathrm{C}$ em estufa de circulação forçada de ar, até atingir peso constante. O material vegetal foi, então, pesado, triturado, e submetido à análise química, para determinação dos teores de P, K, Ca, Mg, Fe, Mn e Al.

Para a determinação das análises químicas, as amostras foram digeridas em uma mistura dos ácidos nítrico e perclórico concentrados, segundo o método descrito por Malavolta et al. (1989). Em seguida, os extratos foram utilizados para a determinação dos teores totais de fósforo, por colorimetria de molibdato-vanadato; potássio, por fotometria de chama e cálcio, magnésio, ferro e manganês por espectrofotometria de absorção atômica. A análise do teor de alumínio foi realizada na Seção de Química Analítica do CENA/USP, através de espectrometria de emissão atômica com plasma induzido de argônio, em aparelho Jarrel Ash, modelo 975. Os dados do experimento foram submetidos à análise de variância, sendo as médias comparadas através do teste de Tukey ao nível de $5 \%$. As análises foram realizadas utilizando-se o programa estatístico SAS (Statistical Analysis System).

\section{RESULTADOS E DISCUSSÃO}

Os efeitos do alumínio e do cálcio na produção de matéria seca da parte aérea e raízes de mudas de pimenta do reino estão apresentados na TABELA 2. Os resultados indicam que houve interação significativa entre os dois fatores. Os dados revelam que houve a inibição progressiva no crescimento das raízes e parte aérea das plantas com o aumento da concentração de alumínio na solução, independente da concentração de cálcio. Verifica-se que, independentemente do nível de cálcio, o alumínio causou aumento do peso da parte aérea até a dose $10 \mathrm{mg}$ $\mathrm{dm}^{-3}$ decrescendo quando a concentração de alumínio foi $20 \mathrm{mg} \mathrm{dm}^{-3}$. O mesmo aconteceu com o peso das raízes até a concentração de cálcio $40 \mathrm{mg} \mathrm{dm}^{-3}$. O efeito do cálcio no peso da parte aérea foi influenciado pelo alumínio.

TABELA 2 - Efeitos da interação alumínio e cálcio na massa da matéria seca da parte aérea e das raizes de mudas de pimenta-do- reino.

\begin{tabular}{|c|c|c|c|c|c|c|}
\hline \multirow{3}{*}{$\begin{array}{c}\text { Doses de } \\
\text { Cálcio }\end{array}$} & \multicolumn{6}{|c|}{ Doses de $\mathrm{Al}\left(\mathrm{mg} \mathrm{L}^{-1}\right)$} \\
\hline & \multicolumn{2}{|c|}{0} & \multicolumn{2}{|c|}{10} & \multicolumn{2}{|c|}{20} \\
\hline & Parte aérea & Raiz & Parte aérea & Raiz & Parte aérea & Raiz \\
\hline$\left(\mathrm{mg} \mathrm{L}^{-1}\right)$ & 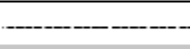 & - & g/pla & - & - & (- \\
\hline 10 & (1) $11,12 \mathrm{aB}$ & $2,06 \mathrm{aB}$ & $20,70 \mathrm{aA}$ & $2,51 \mathrm{aB}$ & $12,03 \mathrm{bC}$ & $1,91 \mathrm{aB}$ \\
\hline 40 & $13,31 \mathrm{aB}$ & 2,55 aA & $17,40 \mathrm{aA}$ & $2,79 \mathrm{aA}$ & $12,86 \mathrm{cB}$ & $2,10 \mathrm{aB}$ \\
\hline 160 & $15,14 \mathrm{aB}$ & 2,76 aA & $16,37 \mathrm{bA}$ & 3,10 aA & $15,15 \mathrm{aA}$ & $2,51 \mathrm{aA}$ \\
\hline
\end{tabular}

(1) Médias seguidas pela mesma letra minúscula nas colunas e maiúscula nas linhas năo apresentam diferença significativas ao nivel de $5 \%$, pelo teste de Tukey.

Segundo Helyar (1978), as doses crescentes de alumínio causam declínio exponencial na produção da parte aérea. Quando se usam espécies mais tolerantes, nota-se um estímulo na produção em baixas doses de alumínio, mas o declínio volta a aparecer em altas doses. Embora o alumínio não seja considerado um elemento essencial, e ainda se desconheçam os mecanismos pelos quais, em baixas concentrações, ele possa, algumas vezes, induzir um aumento no crescimento ou produzir outros efeitos desejáveis, Foy (1974) discute várias possibilidades de explicação para o fato: 1) aumento na disponibilidade do ferro em solos calcários (através da hidrólise do Al e da diminuição do pH); 2) correção ou prevenção de deficiência de ferro, pela liberação do ferro adsorvido em sítios metabolicamente inativos dentro da planta; 3) bloqueio dos sítios, da parede celular, carregados negativamente, promovendo a absorção de fósforo; 4) retardamento da deterioração das raízes em baixas concentrações de cálcio pelo crescimento mais lento; 5) correção ou prevenção do efeito de concentrações excessivas de fósforo; 6) prevenção de toxicidade de cobre e manganês; 7) redução do crescimento indesejável do topo de portaenxertos ricos em nitrogênio.

Na TABELA 3 estão os dados dos efeitos dos tratamentos nas concentrações de cálcio e de alumínio nas raízes da pimenteira do reino. A concentração de cálcio cresceu com o aumento da concentração dele na solução sem alumínio. A adição de alumínio na solução diminuiu progressivamente a absorção de cálcio. Observa-se que não houve efeito do aumento das doses de cálcio na solução de 10 a $160 \mathrm{mg} \mathrm{dm}^{-3}$, na absorção de alumínio pelas raízes da pimenteira do reino. Os dados permitem afirmar que o alumínio inibiu significativamente a absorção de cálcio quando sua concentração foi $40 \mathrm{mg} \mathrm{dm}^{-3}$. Esses resultados alertam para a redução do sistema radicular que terá influência na absorção de água e nutrientes, pela menor área de solo explorada, conforme Foy (1976). Não houve efeito antagônico entre o cálcio e o alumínio, mas sim efeito negativo do alumínio na absorção de cálcio. As raízes apresentavam-se grossas, com aspecto quebradiço, além de coloração amarelo-escura ou marron, e com aspecto coralóide, sem ramificações das laterais conforme descrito também por (Malavolta \& Kliemann, 1985). 
TABELA 3 - Influência dos tratamentos no acúmulo de aluminio e cálcio nas raizes das mudas de pimenta-doreino.

\begin{tabular}{|c|c|c|c|}
\hline \multicolumn{2}{|c|}{ Concentração } & \multicolumn{2}{|c|}{ Conteúdo } \\
\hline $\mathrm{Ca}$ & $\mathrm{Al}$ & $\mathrm{Ca}$ & $\mathrm{Al}$ \\
\hline \multicolumn{2}{|c|}{ - } & \multicolumn{2}{|c|}{--- mg planta-1_- } \\
\hline \multirow{3}{*}{10} & 0 & (1) $5,16 \mathrm{a}$ & $0,56 \mathrm{f}$ \\
\hline & 10 & 3,79 a & 0,99 ef \\
\hline & 20 & $3,08 \mathrm{a}$ & $6,51 \mathrm{a}$ \\
\hline \multirow{3}{*}{40} & 0 & 7,57 a & $0,43 \mathrm{f}$ \\
\hline & 10 & $5,39 \mathrm{~b}$ & $4,46 \mathrm{bc}$ \\
\hline & 20 & $5,10 \mathrm{~b}$ & $5,33 a b$ \\
\hline \multirow{3}{*}{160} & 0 & $8,29 a$ & $0,41 \mathrm{f}$ \\
\hline & 10 & 7,94 a & 2,06 de \\
\hline & 20 & $6,18 \mathrm{a}$ & $3,30 \mathrm{~cd}$ \\
\hline
\end{tabular}

(1) Médias seguidas pela mesma letra minúscula nas colunas $e$ maiúscula nas linhas năo apresentam diferença significativas ao nivel de $5 \%$, pelo teste de Tukey.

As considerações de Marschner (1986), segundo os quais o alumínio pode inibir a absorção de cálcio, principalmente como resultado do bloqueio ou competição nos sítios de troca, justificam o pequeno efeito do alumínio, observado no presente estudo. Do mesmo modo, Foy (1984) verificou que a toxicidade de Al pode se manifestar como uma deficiência de $\mathrm{Ca}$ induzida, em consequência de redução do transporte do nutriente na planta, provocando um colapso nos pontos de crescimento em valores de $\mathrm{pH}<5,5$. O antagonismo Al x Ca talvez seja o fator mais limitante na absorção de cálcio.

Os dados analíticos referentes aos teores de fósforo, potássio, cálcio, magnésio, alumínio, ferro e manganês nas folhas para diagnóstico, em função das doses de $\mathrm{Al}$ e $\mathrm{Ca}$, são apresentados na TABELA 4. Os teores de alumínio aumentaram progressivamente de acordo com os incrementos de sua concentração na solução nutritiva. Em geral, o alumínio adicionado na solução fez decrescer os teores de fósforo, potássio, cálcio, magnésio, ferro e manganês e aumentar a concentração de alumínio.

TABELA 4 - Efeito dos tratamentos com alumínio e cálcio na composição química das folhas da pimenteira do reino amostradas cinco mêses após o início dos tratamentos.

\begin{tabular}{|c|c|c|c|c|c|c|c|c|}
\hline \multicolumn{3}{|c|}{ Doses } & \multicolumn{6}{|c|}{ Teor nas folhas } \\
\hline $\mathrm{Ca}$ & $\mathrm{Al}$ & $\mathrm{P}$ & $\mathrm{K}$ & $\mathrm{Ca}$ & $\mathrm{Mg}$ & $\mathrm{Al}$ & $\mathrm{Fe}$ & $\mathrm{Mn}$ \\
\hline \multicolumn{2}{|c|}{--- mg L-1 -..- } & \multicolumn{4}{|c|}{ - g kg-1 - } & \multicolumn{3}{|c|}{ - } \\
\hline \multirow{3}{*}{10} & 0 & $1,60 \mathrm{a}$ & $14,20 \mathrm{a}$ & $5,03 \mathrm{a}$ & 4,36 a & $107,22 \mathrm{c}$ & $352,77 a$ & $190,11 \mathrm{a}$ \\
\hline & 10 & $1,33 \mathrm{ab}$ & $14,03 \mathrm{a}$ & $3,36 \mathrm{~b}$ & 4,40 a & $129,81 \mathrm{~b}$ & $228,67 a$ & $128,91 \mathrm{~b}$ \\
\hline & 20 & $1,10 \mathrm{~b}$ & $11,60 \mathrm{~b}$ & $2,03 \mathrm{c}$ & $2,26 b$ & 165,89 a & $226,26 a$ & $82,76 \mathrm{c}$ \\
\hline \multirow{3}{*}{40} & 0 & $1,56 \mathrm{a}$ & 17,76 a & $3,50 \mathrm{a}$ & 3,66 a & $90,38 \mathrm{c}$ & 290,77 a & 122,82 a \\
\hline & 10 & $1,13 b$ & $13,26 \mathrm{~b}$ & 3,36 a & $3,40 \mathrm{a}$ & $140,18 b$ & $167,50 \mathrm{~b}$ & $106,19 b$ \\
\hline & 20 & $0,90 \mathrm{a}$ & $7,66 \mathrm{c}$ & $3,10 \mathrm{a}$ & $2,56 \mathrm{~b}$ & $170,85 \mathrm{a}$ & $80,73 \mathrm{c}$ & $66,62 \mathrm{c}$ \\
\hline \multirow{3}{*}{160} & 0 & $1,56 \mathrm{a}$ & 19,20 a & 12,63 a & $4,03 \mathrm{a}$ & $78,26 \mathrm{c}$ & 246,39 a & 129,15 a \\
\hline & 10 & $1,33 \mathrm{~b}$ & $9,76 \mathrm{~b}$ & $7,63 \mathrm{~b}$ & $3,26 \mathrm{~b}$ & $99,02 \mathrm{~b}$ & $142,32 \mathrm{~b}$ & 128,95 a \\
\hline & 20 & $1,00 \mathrm{~b}$ & $2,30 \mathrm{c}$ & $4,20 \mathrm{c}$ & $1,73 \mathrm{c}$ & $188,66 a$ & $114,28 \mathrm{~b}$ & $67,62 \mathrm{~b}$ \\
\hline
\end{tabular}

(1)Médias seguidas pela mesma letra minúscula nas colunas e maiúscula nas linhas năo apresentam diferença significativas ao nivel de $5 \%$, pelo teste de Tukey.

A diminuição desses nutrientes pode ser atribuída ao efeito direto de alumínio na absorção de $\mathrm{P}, \mathrm{Ca}$ e $\mathrm{Mg}$, concordando com os resultados publicados por Foy (1974). Portanto, os resultados obtidos no presente experimento, acerca dos efeitos do alumínio na absorção de cálcio, podem ser importantes na indução da deficiência de cálcio e, consequentemente, na diminuição da produção de pimenta do reino.

\section{CONCLUSÕES}

Houve efeito positivo do alumínio na produção de matéria seca da parte aérea e raízes até a dose de $10 \mathrm{mg} \mathrm{dm}^{-3}$, tendo tido a maior produção nesta dose.

- A adição de $20 \mathrm{mg} \mathrm{dm}^{-3}$ de Al diminuiu o peso da matéria seca e o teor de nutrientes o qual foi associado como início do efeito da toxicidade, esperado-se maior intensificação ao longo do tempo.

- A absorção de cálcio pelas raízes da pimenteira do reino foi reduzida com o aumento da concentração de alumínio na solução.

- O aumento da concentração de cálcio em solução não diminuiu a toxicidade do alumínio nas plantas. 
ALBUQueRQUe, F.C.; VELOSO, C.A.C.; DUARTE, M.L.R.; KATO, O.R. Pimenta do reino; recomendações básicas para seu cultivo. Belém: EMBRAPA, UEPAE, 1989. 40p. (Documentos, 12).

CAMBRAIA, J. Mecanismos de tolerância a toxidez de alumínio em plantas. In: REUNIÃO BRASILEIRA DE FISIOLOGIA VEGETAL, 2., Piracicaba,1989. Anais. Piracicaba: SBFV/ESALQ, 1989. p.85-92.

FOY, C.D. Soil chemical factors limiting plant root growth In: HATFIELD, J.L.; STEWART, B.A. Limitations to plant root growth. New York: Springer-Verlag, 1992. p.97-149.

FOY, C.D. Physiological effects of hydrogen, aluminum and manganese toxicities in acid soil. In: ADAMS, F. (Ed.) Soil acidity and liming. 2.ed. Madison: Soil Science Society American, 1984. p.57-97.

FOY, C.D.; CHANEY, R.L.; WHITE, M.C. The physiology of metal toxicity in plants. Annual Review of Plant Physiology, v.29, p.511-566, 1978.

FOY, C.D. Differential aluminum and manganese tolerances of plant species and varieties in acid soils. Ciência e Cultura, v.28, p.150-155, 1976.

FOY, C.D. Effect of aluminum on plant growth. In: CARSON, F.W. (Ed.) The plant root and its environment. Charlottesville: University Press of Virginia, 1974. p.601-642.

FOY, C.D.; FLEMING, A . L.; ARMIGER, W.H. Aluminum tolerance of soybean varieties in relation to calcium nutrition. Agronomy Journal, v. 61, p.505-511, 1969.

HELYAR, K.R. Effects of aluminum and manganese toxicity on legume growth. In: ANDREW, C.S.; KAMPRATH, E.J. (Ed.) Mineral nutrition of legumes in tropical an subtropical soils. Melbourne: CSIRO, 1978. p.207-231.

LOPES, A.S. Solos sob "cerrado" ; características, propriedades e manejo. Piracicaba: Potafós, 1984. 162p.

MALAVOLTA, E.; VITTI, G.C.; OLIVEIRA, S.A. Avaliação do estado nutricional das plantas; princípios e aplicações. Piracicaba: Potafós, 1989. 201p.

MALAVOLTA, E.; KLIEMANN, H.J. Desordens nutricionais no cerrado. Piracicaba: Potafós, 1985. 136p.

MARSCHNER, H. Mineral nutrition of higher plants. London: Academic Press, 1986. 674p.

PAVAN, M.A.; BINGHAM, F.T. Toxidez de alumínio em cafeeiros cultivados em solução nutritiva. Pesquisa Agropecuária Brasileira, v.17, p.1293-1302, 1982.

PAVAN, M. A . Efeitos tóxicos de alumínio em mudas de cafeeiro em relação à nutrição de cálcio. Revista Brasileira de Ciência do Solo, v.6, p.209-213, 1982.

PRATT, P.F. Aluminum. In: CHAPMAN, H.D. (Ed.) Diagnostic criteria for plants and soils. Berkeley: University of California,1966. p.3-12.

VELOSO, C.A.C.; MURAOKA, T.; MALAVOLTA, E.; CARVALHO, J.G. de. Efeitos do alumínio em pimenteira do reino (Piper nigrum, L.). Scientia Agricola, v.52, n.2, p.368-375, 1995.

WAARD, P.W.F. Foliar diagnosis, nutrition and yield stability of black pepper (Piper nigrum,L.) in Sarawak. Amsterdam: Royal Tropical Institute, 1969. 149p. (Communication, 58).

Recebido em 18.09.98

(cc)EY-NC Todo o conteúdo deste periódico, exceto onde está identificado, está licenciado sob uma Licença Creative Commons

Scientia Agricola

USP/ESALQ - Scientia Agricola

Av. Pádua Dias, 11

13418-900 Piracicaba SP Brazil

Tel.: +55 19 3429-4401/3429-4486

Fax: +55 19 3429-4401

efMail

scientia@esalq.usp.br 